\title{
Editorial
}

\section{The impacts of emerging management support systems}

\author{
Efraim TURBAN and Paul R. WATKINS \\ Inst. of Safety and Systems Management, University of Southern \\ California, Los Angeles, CA 90089-0021, USA
}

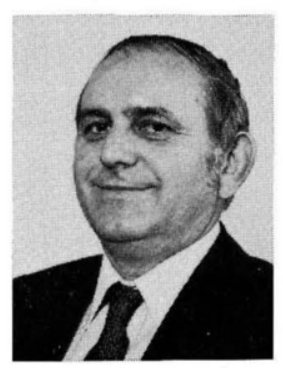

Efraim Turban, Professor of Systems Science and Director of Information Systems Research Laboratory, including Expert and Decision Support Systems, at the University of Southern California, is interested in emerging computer technologies and their organizational and managerial impacts. $\mathrm{He}$ is the author of several books, including Decision Support Systems and Expert Systems, and Fundamentals of Management Science. $\mathrm{He}$ also authored about 50 papers which appeared in the major professional journals. Graduated from the University of California at Berkeley (MBA, Ph.D.), Dr. Turban held faculty appointments at Lehigh University and Florida International University. He has held a visiting appointment at UCLA. Dr. Turban worked in industry as industrial engineer and management consultant with leading corporations, such as General Electric. He has been a consultant to many corporations and governments.

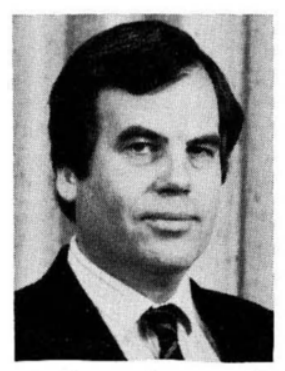

Paul R. Watkins is an Associate Professor at the University of Southern California and director of the Expert Systems Program in the School of Accounting. His research interests are in the areas of judgment and decision making and judgment support systems (Expert Systems, Artificial Intelligence). His research has been published in a variety of accounting, systems and operations research journals and he has made numerous presentations at national and international meetings of accounting, information systems and management science symposia. He has received major competitive research grants from various research foundations and was recently awarded the TIMS Roundtable Competitive Research award for research in Artificial Intelligence and Management (with E. Turban). He serves on the editorial boards of several journals and is a referee for various scholarly societies and research journals. He provides consulting advice for major international firms in the areas of applied Expert Systems.

North-Holland

Human Systems Management 7 (1987) 7-10
This editorial provides an overview of two emerging technologies that have wide implications for decision making and organizational issues. These technologies are decision support systems (DSS) and expert systems (ES). Both technologies are defined and discussed as stand-alone systems and as integrated systems. A perspective is provided to enable better integration of the papers that follow in this special issue.

Keywords: Expert systems, artificial intelligence, decision support systems, information technologies, intelligent systems, knowledge-based systems.

The information revolution has already impacted many organizations. This impact varies in coverage and intensity. In some organizations business is conducted as though computers do not exist. In others, everything has been changed; from job content to allocation of power.

Initially computers were used to improve organizational efficiency and to increase the quality of services and products. At that time most of the systems dealt with transactions (or data) processing. During the 60's a new dimension was added, management information systems (MIS). This computer-based system intended to receive, extract and integrate data from various sources, in order to provide timely information needed for managerial decision making.

MIS has been most successful in providing information for routine, structured, and anticipated types of decisions. In addition, they have been successful in acquiring and storing large quantities of detailed data concerning transaction processing. MIS have been less successful in supporting complex decision situations. This is due to a lack of capabilities necessary for such support, and MIS traditionally have not facilitated easy development or even ease of use by managers. Furthermore, as managers climb the corporate 
ladder, they must increasingly deal with matters outside the organization. Most MIS are built with a focus on the internal organization. Hence, the support of the traditional MIS decreases as the organizational level increases.

The concept of decision support systems was developed in the early 70's in an attempt to rectify some of the shortcomings of the MIS of the 60's. It also intended to help in reducing the waiting line (backlog) of requests for computers systems' developments. In the late 70's the discipline of artificial intelligence started to move into commercialization (see special issue of $H S M$, Vol. 5, no. 2, 1985). Expert systems (ES) is becoming a most promising area in AI.

The microcomputer revolution, end-user computing, availability of friendly software development tools and recent advances in telecommunications, have greatly accelerated the use of DSS and ES during the last few years. For example, Walker and Miller [5] describe over 1000 commercial expert systems that existed at the end of 1985. Of this list, about 900 were developed in 1984 and 1985.

Before discussing the potential organizational impacts of DSS and ES, let us briefly describe these tools and list their major characteristics and benefits.

Decision support systems: The concepts involved in DSS were first articulated in the early 1970's by Scott-Morton under the term 'management decision systems'. He defined such systems as 'interactive computer-based systems, which help decision makers utilize data and models to solve unstructured problems' [3].

Another classical definition of DSS, provided by Keen and Scott-Morton [1], follows:

'Decision support systems couple the intellectual resources of individuals with the capabilities of the computer to improve the quality of decisions. DSS is computer-based support for management decision makers who deal with semi-structured problems.'

The foregoing definition indicates the four major characteristics of DSS:

- DSS incorporate both data and models.

- They are designed to assist managers in their decision processes in semi-structured or unstructured tasks.

- They support, rather than replace, managerial judgment.

- The objective of DSS is to improve the effectiveness of the decisions, not the efficiency with which decisions are made.

Additional characteristics and benefits which were recognized over the last fifteen years are:

(a) Ability to support the solution of complex problems. A DSS enables the solution of complex problems that ordinarily cannot be solved by other computerized approaches (or can be solved at a much slower pace).

(b) Fast response to unexpected situations which result in changed input. A DSS enables a thorough, quantitative analysis in a very short time. Even major changes in a scenario can be evaluated objectively in a timely manner.

(c) Ability to try several different strategies under different configurations, quickly and objectively. For example, a complete 'what-if' analysis was carried out to examine the downside risk of a joint venture at Houston Minerals Corp. This analysis, which took 20 minutes, would have taken days or weeks with other computerized systems.

(d) New insights and learning. The user can be exposed to new insights through the composition of the model and an extensive sensitivity 'what-if' analysis. The new insights can help in training inexperienced managers and other employees as well.

(e) Facilitated communication. Data collections and model construction experiments are being executed with active users' participation; thus greatly facilitating communication among managers. The objectivity and logic of the decision process can make employees more supportive of organizational decisions. The 'what-if' analysis can be used to satisfy skeptics in turn improving teamwork.

(f) Improved management control and performance. DSS can increase management control over expenditures and improve performance of organizations.

(g) Cost savings. Routine applications of a DSS may result in considerable cost reduction, or 
in reducing (or eliminating) the cost of wrong decisions.

(h) Objective decisions. The decisions derived from DSS's are more consistent and objective than complex decisions that are made intuitively. Also, they are based on a thorough analysis and are executed with greater participation of the individuals who are affected by the decisions. Therefore, the decisions are of a better quality and have a greater chance of success.

(i) Improving managerial effectiveness. All of the above capabilities can improve managerial effectiveness (and personal efficiency) by allowing managers to perform a task in a way that uses less time or less effort. The DSS provides managers with more 'quality time' for analysis, planning and implementation by reducing the need for number crunching.

(j) Support to individuals and/or groups. DSS can be used to support individual managers and/or groups of managers.

Expert systems: When an organization has a complex decision to make or problem to solve, it often turns to experts for advice. These experts have specific knowledge and experience in the problem area. They are aware of the alternatives available, the chances of success, and costs the business may incur. Companies engage experts for advice on such matters as: computer acquisitions, mergers and acquisitions, or advertising strategy. The more unstructured the situation, the more expensive is the advice. Expert systems are an attempt to mimic the human experts. Typically, an expert system is a decision-making and/or problem-solving package of computer hardware and software that can reach a level of performance comparable to, or even exceeding that of, a human expert in some specialized and usually narrow problem areas.

The basic idea behind ES is simple. Expertise, which is the vast body of task-specific knowledge, is transferred from the human to a computer. This knowledge is then stored in the computer. The user calls upon the computer for specific advice as it is needed. The computer can make inferences and arrive at a specific conclusion. Then, like a human consultant, it will advise the nonexperts and explain, if necessary, the logic behind the advice.
Expert systems can provide organizations with improved productivity levels and increased competitive advantages due to the following potential benefits:

(a) Monetary savings. Human expertise is usually very expensive compared to a frequently used expert system.

(b) Improved quality. Some ES have proved to do a better job than humans. They make fewer mistakes and are more consistent in their recommendations.

(c) ES are compatible with managers' decision styles. ES are based on judgment, which is extensively used by managers.

(d) ES can be used as a training vehicle. They can be used to train non-experts and for improving the expertise of experts.

(e) ES can free experts from time-consuming, routine tasks. Many experts are too busy advising non-experts on what to do. ES can free experts from repetitive advice and training, or from data search tasks, and enable them to concentrate on more creative tasks.

The characteristics outlined above clearly point to the significant potential impacts on organizations. These impacts can completely change organizations and the way that people operate in them. Already major changes are planned in financial institutions, such as banks and insurance companies, where DSS and ES are expected to show considerable payoffs. Changes are expected to occur in almost any facet of these organizations, ranging from structure to job content.

The organizational impacts on DSS and ES will not occur over night; some of them will go through a lengthy evolutionary process. These systems tend to develop slowly and expand over a long time. Furthermore, DSS and ES may not be stand-alone systems. They may be integrated with already existing computer-based information systems [4]. All this may make it difficult to isolate and assess the impacts of these technologies.

Despite the difficulties in assessing the organizational impacts it is necessary to do so. There are some predictions that DSS and ES could dominate the MIS field before the year 2000. In such a case 
the impacts could be felt in entire industries and society as a whole (e.g., see Nilsson [2]).

What we are driving at is the need for pure and applied research in the area of organizational impacts of DSS and ES. Several of the issues involved are presented in this special issue of $\mathrm{Hu}$ man Systems Management. This editorial paper is intended to set the stage for the forthcoming papers by briefly defining the new technologies and describing their major characteristics. It is also intended to call attention to the magnitude of the impacts which we believe may be extremely large in many organizations.

The papers in this special issue represent both theoretical and empirical work of respected researchers and observers of the DSS and ES scene. They cover only a small portion of much broader areas. Yet, they deal with large enough diversity of topics and issues which we believe could be of great interest to the reader and could ignite additional research in the future.

\section{References}

[1] Keen, P.G.W. and N.S. Scott-Morton, Decision support systems, an organizational perspective (Addison-Wesley, Reading, MA, 1978).

[2] Nilsson, N.J., Artificial intelligence, employment, and income, Human Systems Management 5 (1985) 123-135.

[3] Scott-Morton, M.S., Management decision systems: Computer based support for decision making, Division of Research, Harvard University, Cambridge, MA (1971).

[4] Turban, E. and P.R. Watkins, A framework for integration of ES and DSS, MIS Quarterly (June, 1986) 120-136.

[5] Walker, T.C. and R.K. Miller, Expert systems 1986, an assessment of technology and applications (SEAI Publications, Madison, GA, 1986). 